\title{
9 \\ El primer concurso de talentos online de España como práctica innovadora e interdisciplinar en los estudios de Comunicación durante la pandemia
}

\author{
Spain's first online talent contest as an innovative and \\ interdisciplinary practice in Communications Studies during the \\ pandemic
}

\author{
Dra. Rocío Gago Gelado \\ Universidad Nebrija (Madrid) \\ mgago@nebrija.es \\ ORCID: https://orcid.org/0000-0003-4024-1143
}

Lic. Andy Damián Tavárez Pérez

Universidad Nebrija (Madrid)

atavarez@nebrija.es

ORCID: https://orcid.org/0000-0003-2721-2444

Recibido: 26 de febrero de 2021.

Aceptado: 11 de marzo de 2021.

Publicado: 31 de mayo de 2021.

\author{
Dra. Marta Saavedra Llamas \\ Universidad Nebrija (Madrid) \\ msaavedr@nebrija.es \\ ORCID: https://orcid.org/0000-0002-7762-9996
}

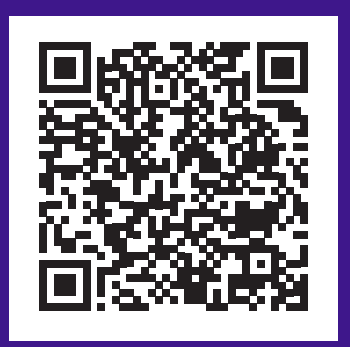

Videopresentación

\section{(c) (†) $\odot$ Esta obra está bajo una licencia internacional Creative}

DOI: https://doi.org/10.21555/rpc.v0i1.2356

Cómo citar: Gago, R. ., Saavedra, M. ., \& Tavárez, A. D. . (2021). Primer concurso de talentos online de España como práctica innovadora e interdisciplinar en los estudios de Comunicación durante la pandemia. RPC, (1), 116-127. https://doi. org/10.21555/rpc.voi1.2356

Revista Panamericana de Comunicación, Año 3, N. 1, enero-julio 2021, pp. 116-127. 


\section{RESUMEN}

Nebrija Medialab es una plataforma de medios que cuenta con cinco laboratorios: Actualidad, Radio, TV, Publicidad y Diseño. El Laboratorio de TV realiza proyectos audiovisuales de información, ficción y entretenimiento para que los alumnos entrenen sus competencias profesionales. Con una metodología ABP, surge en 2018 Nebrija Talent, un concurso de talentos donde los alumnos trabajan delante y detrás de la cámara. Son los estudiantes los que, por un lado, participan demostrando su arte $y$, por otro, organizan el show. Divididos en equipos, atienden las fases de casting, guion, presentación, dirección, realización, cámara, sonido, iluminación, edición y, en colaboración con los laboratorios de Publicidad y Diseño, promoción y gestión de redes sociales. Nebrija Talent vivió el 8 de mayo de 2020 su tercera edición y tuvo que adaptase a la situación impuesta por la COVID-19. Se celebró en formato telepresencial, convirtiéndose en el primer talent show online de España y potenciando, además de la adquisición de las competencias profesionales, la integración creativa y provechosa de las tecnologías y las habilidades digitales entre el alumnado. El programa fue emitido simultáneamente en BlackBoardUltra y YouTube e integró los trabajos de distintos grados del ámbito de la Comunicación y las Artes, significando un modelo de interdisciplinariedad. Este artículo explica la práctica docente y recoge una investigación adhoc acerca de los resultados de aprendizaje alcanzados.

Palabras clave: talent show, competencias profesionales, enseñanza telepresencial, aboratorios universitarios, ABP, COVID-19

\section{ABSTRACT}

Nebrija Medialab is a media platform with five laboratories: News, Radio, TV, Advertising and Design. The TV Lab produces audiovisual projects of information, fiction and entertainment for students to train their professional skills. With a PBL methodology, in 2018 Nebrija Talent Show was created, a talent contest where students work in front of and behind the camera. It is the students who, on the one hand, participate by demonstrating their art and, on the other, organise the show. Divided into teams, they attend to the phases of casting, script, presentation, direction, production, camera, sound, lighting, editing and, in collaboration with the Advertising and Design laboratories, promotion and management of social networks. Nebrija Talent experienced its third edition on 8 May 2020 and had to adapt to the situation imposed by COVID-19. It was held in telepresence format, becoming the first online talent show in Spain and promoting, in addition to the acquisition of professional skills, the creative and profitable integration of technologies and digital skills among students. The programme was broadcast simultaneously on BlackBoardUltra and YouTube and integrated the work of different degrees in the field of Communication and the Arts, representing a model of interdisciplinarity. This article explains the teaching practice and collects ad-hoc research on the learning outcomes achieved.

Keywords: talent show, professional competences, telepresence teaching, university laboratories, PBL, COVID-19 


\section{INTRODUCCIÓN}

$\mathrm{N}$ ebrija Medialab es un proyecto de innovación docente, creado en el curso 2013/14 en la Facultad de Comunicación y Artes de la Universidad Nebrija (Madrid, España). Es un espacio experiencial de aprendizaje, un laboratorio de medios donde los estudiantes entrenan sus competencias profesionales junto a sus docentes y colaboran en proyectos reales de comunicación.

Bebe del estudio Situación actual y perspectiva de futuro de los estudios de Ciencias de la Comunicación (2012), que muestra las competencias demandadas por los empleadores. Indica que el mercado valora la capacidad de autoformación, las destrezas digitales, el espíritu creativo e innovador, la proactividad, curiosidad, inquietud, emprendimiento, trabajo en equipo, correcta comunicación, visión analítica y planificación. Asimismo, se han ido actualizando sus objetivos con estudios sobre perfiles en el sector de la comunicación como los de Besalú-Casademont, Schena y Sánchez-Sánchez (2017), Saavedra, Grijalba y Pedrero (2018), Civil i Serra (2018), Miguel (2018) o Saavedra, Herrero y CastiIlo (2020).

Nebrija Medialab trata de favorecer la formación de los alumnos y, al mismo tiempo, les ofrece vivencias reales preprofesionales para ir aglutinando méritos que hagan diferente su currículum en un proceso de selección. No sólo se practican las competencias específicas, sino también transversales como el trabajo en equipo, el liderazgo, la toma de decisiones, la motivación o la innovación.

A nivel general, el proyecto se estructura con una metodología ABP, aprendizaje basado en proyectos. Según Jones, Rasmussen, y Moffitt (1997; en Sánchez, 2013).

El aprendizaje basado en proyectos es un conjunto de tareas de aprendizaje basada en la resolución de preguntas y/o problemas, que implica al alumno en el diseño y planificación del aprendizaje, en la toma de decisiones y en procesos de investigación, dándoles la oportunidad para trabajar de manera relativamente autónoma durante la mayor parte del tiempo, que cul- mina en la realización de un producto final presentado ante los demás.

Así, se centra el aprendizaje en el alumno, promoviendo la motivación intrínseca, estimulando el aprendizaje colaborativo, impulsando la mejora continua y multiplicando el compromiso del estudiante (Martí, 2010). En nuestro laboratorio de medios se requiere que el estudiante realice un producto, participe en un reto común. Ken Bain (2009) defiende que esta metodología ofrece la posibilidad de adquirir competencias en un proyecto experiencial que no sólo reta a los estudiantes, sino que motiva a los docentes.

Por su parte, Johnson, Johnson y Smith (2006) recuerdan que esta metodología provoca un escenario de aprendizaje motivador y que, ante la solución de un problema o reto común, el equipo desarrolla nuevas habilidades y se activa el pensamiento profesional. Sigue un enfoque constructivista y mientras el alumno se sitúa en el centro del aprendizaje, el profesor pasa a ser un guía, por lo que las relaciones se estrechan y el ambiente de trabajo es auténtico.

Desde su implantación, Medialab ha ido creciendo en número de laboratorios, proyectos y estudiantes. En el curso 2019/20 se alcanzó a 70 estudiantes, de 9 grados diferentes, lo que potencia la interdisciplinariedad.

Los laboratorios se estructuran así:

» Actualidad: es el responsable de la redacción de la revista [N]. Se trata de una publicación semestral y registrada (M-37777-2019), cuyo target es el estudiante universitario.

» Radio: ejecuta una programación semanal y forma parte de la Asociación de Radios Universitarias de España.

» Diseño: maqueta la revista, idea creatividades y proyectos de identidad gráfica.

» Publicidad: realiza proyectos de comunicación y marca para clientes reales. 
» Televisión: desarrolla proyectos audiovisuales de información, ficción y entretenimiento.

Este último laboratorio acoge el proyecto contenido en este artículo porque dentro de las actividades desarrolladas, se localiza Nebrija Talent.

Con una metodología ABP, en diciembre de 2017 realizamos la I Edición de Nebrija Talent: un concurso de talentos donde los alumnos trabajan delante y detrás de la cámara. Se trataba de crear un espacio real de aprendizaje, imitando la realización de un programa de televisión de talentos; así, los alumnos practicaban los roles profesionales de una producción audiovisual, los dictámenes del formato de entretenimiento y, además, demostraban sus habilidades artísticas. El programa se grabó en el plató de televisión del Campus de Princesa y los alumnos organizaron el show desde su fase de preproducción hasta la postproducción.

En el curso 2018/19, la II Edición de Nebrija Talent creció: aumentó la duración, se cambió de plató para buscar un espacio mayor que ofreciera más elementos narrativos a los guionistas, se ampliaron los medios técnicos y el equipo de trabajo. Además, la participación como concursante se hizo extensiva a estudiantes de todas las facultades, favoreciendo el conocimiento de Medialab fuera de la Facultad de Comunicación y Artes, ampliando la experiencia de aprendizaje y creando lazos entre estudiantes de distintas disciplinas.

La COVID-19 hizo que los responsables del proyecto lo adaptaran a las circunstancias y, en vez de suspenderse, la III Edición de Nebrija Talent volvió a tener como ingredientes la innovación, la interdisciplinariedad a lo que se unía la telepresencialidad.

Lejos de romperse la interacción necesaria en los proyectos $A B P$, los entornos remotos activan la comunicación multicanal y es que, como sostienen Llorens-Largo, Villagrá-Arnedo, Gallego-Durán y Molina-Carmona (2021), esta metodología se ha adaptado muy bien al confinamiento ya que prepara a los estudiantes para ser más autónomos. Además, el seguimiento online produce una interacción directa y el control del proceso de aprendizaje y las herramientas digitales ayudan a la colaboración de los equipos.
Los docentes pudieron trabajar no solo las competencias específicas, sino que se sumaron habilidades tecnológicas. Como apunta Sales, Cuevas-Cerveró y Gómez Hernández (2020), aunque el alumnado está habituado a la tecnología, tiene un déficit de competencias informacionales y digitales.

Partimos de una hipótesis de corte afirmativo que sostiene que los proyectos docentes asentados en una metodología APB se pueden desarrollar con éxito en un escenario de educación a distancia. Así, el objetivo general del presente artículo es analizar el caso de éxito de la III Edición de Nebrija Talent y relatar su adaptación al formato telepresencial.

Los objetivos específicos fueron: procurar un espacio de trabajo real; ofrecer a los alumnos un proyecto de comunicación donde pudieran entrenar sus competencias; ejercer los roles profesionales contenidos en una producción de televisión; adaptar la metodología de aprendizaje a un entorno virtual y seleccionar las herramientas digitales más adecuadas para alcanzar los resultados de aprendizaje, ampliando los mismos con la adquisición de hábitos de teletrabajo y de colaboración en entornos virtuales.

\section{METODOLOGÍA}

La primera parte de esta investigación se asienta sobre un estudio descriptivo para, como hemos visto, explicar el proyecto Nebrija Medialab, así como su división de televisión, y justificar las metodologías docentes empleadas. Indicamos las ventajas del método ABP, así como las claves de su adaptación a escenarios remotos.

La segunda parte del estudio analiza el caso de éxito de la III Edición de Nebrija Talent, proyecto docente que desarrolla las competencias profesionales de los perfiles vinculados al entretenimiento televisivo.

Ejecutaremos esta parte en dos etapas:

» Primero, analizaremos el caso siguiendo un patrón de observación inspirado en Llorens-Largo, Villagrá-Arnedo, Gallego-Durán y Molina-Carmona (2021), que precisamente examinan la 
metodología ABP durante el confinamiento. Recoge los ítems:

» Descripción del proyecto docente y participantes

» Organización, planificación y desarrollo

» Adaptación a la docencia remota

» Herramientas tecnológicas utilizadas

» Evaluación

Segundo, completaremos el estudio con una investigación de tipo cualitativo ad hoc realizada bajo la técnica del focus group, que permite la expresión de distintas posturas y actitudes por parte de los participantes, el intercambio de información y la orientación del discurso sobre la realidad a investigar (Canales y Peinado, 1995).
La variable concurrente entre los participantes será su intervención en el proyecto. Se considera importante, además, que los participantes hayan intervenido en más de una edición para poder valorar la adaptación al modelo telepresencial; esta segunda variable no se respeta en los alumnos de primer curso. Procuraremos la representación de hombres y mujeres y la presencia de alumnos de diferentes titulaciones al tratarse de un proyecto interdisciplinar. Asimismo, incorporaremos a perfiles de distintos cursos para recoger el máximo espectro de estudiantes convocados.

La muestra cualitativa no busca una representatividad estadística sino estructural, puesto que lo que pretende comprender son los distintos sentidos sociales y las relaciones con el fenómeno investigado (Rubio y Perlado, 2015).

Tabla 1. Participantes en el focus group

\begin{tabular}{|c|c|c|c|c|c|c|c|c|}
\hline & GRADO & CURSO & EDAD & SEXO & $\begin{array}{l}\text { PARTICIPACIÓN EN LA III } \\
\text { EDICIÓN DE NEBRIJA } \\
\text { TALENT }\end{array}$ & $\begin{array}{l}\text { PARTICIPACIÓN EN } \\
\text { EDICIONES ANTERIORES }\end{array}$ & $\begin{array}{l}\text { ROL EJECUTADO } \\
\text { EN EL PROYECTO }\end{array}$ & $\begin{array}{l}\text { CóDIGO } \\
\text { MIEMBRO }\end{array}$ \\
\hline$\vec{P}$ & $\begin{array}{l}\text { Comunicación } \\
\text { Audiovisual }\end{array}$ & $4^{\circ}$ & 25 & $\mathrm{H}$ & Sí & Sí & Dirección & E1 \\
\hline 을 & Periodismo & $2^{\circ}$ & 20 & $\mathrm{H}$ & Sí & Sí & Producción & E2 \\
\hline$\frac{0}{11}$ & Periodismo & $2^{\circ}$ & 19 & $M$ & Sí & Sí & Guion & E3 \\
\hline$\stackrel{1}{\rightleftarrows}$ & Artes Escénicas & $3^{\circ}$ & 20 & $M$ & Sí & Sí & Guion & E4 \\
\hline II & $\begin{array}{l}\text { Comunicación } \\
\text { Audiovisual }\end{array}$ & $1^{\circ}$ & 20 & M & Sí & No & Guion & E5 \\
\hline 를 & Publicidad & $1^{\circ}$ & 18 & $M$ & Sí & No & Promoción & E6 \\
\hline$\frac{0}{2}$ & Diseño Digital & $2^{\circ}$ & 20 & $\mathrm{H}$ & Sí & Sí & Creatividad & E7 \\
\hline$\underline{2}$ & $\begin{array}{l}\text { Comunicación } \\
\text { Audiovisual }\end{array}$ & $2^{\circ}$ & 21 & $\mathrm{H}$ & Sí & Sí & Creatividad & E8 \\
\hline$\frac{Z}{7}$ & $\begin{array}{l}\text { Comunicación } \\
\text { Audiovisual }\end{array}$ & $1^{\circ}$ & 18 & $\mathrm{H}$ & Sí & No & Creatividad & E9 \\
\hline & $\begin{array}{l}\text { Comunicación } \\
\text { Corporativa }\end{array}$ & $1^{\circ}$ & 18 & $M$ & Sí & No & Redes & E10 \\
\hline
\end{tabular}

Fuente: Elaboración propia. 
Estas preguntas guiaron la conversación:

» ¿Cómo valora esta actividad académica y su adaptación al formato telepresencial?

» ¿Qué supuso su participación durante el confinamiento?

» ¿Qué competencias específicas ha practicado? ¿Qué habilidades transversales se han estimulado?

" ¿Se han potenciado otros conocimientos propios de los entornos virtuales de trabajo?

» ¿Se ha resentido la interacción y seguimiento del proyecto?

" ¿Considera que las metodologías activas de enseñanza favorecen la adquisición de los resultados de aprendizaje y que pueden adaptarse al entorno virtual?

\section{RESULTADOS}

\subsection{Evaluación de la adaptación de la actividad docente al formato online}

\subsubsection{Descripción del proyecto docente y participantes}

Bain (2009) explica que para que los proyectos activos de enseñanza, como los impulsados con la metodología ABP, resulten eficaces, los docentes deben:

Crear un entorno para el aprendizaje donde el alumno encuentre las destrezas, actitudes e informaciones que están intentando aprender inmersas en preguntas y tareas, que pudieran ser encontradas en el entorno profesional futuro.

En este sentido, la propia configuración del talent show supone la creación de un entorno real de aprendizaje y el reto de su desarrollo genera en los equipos de trabajo diferentes tareas.
Se establecen ocho departamentos: Dirección, encargado de crear el formato audiovisual; Producción, donde se gestiona la logística del programa y, en este caso, la participación de los concursantes; Guion, ideación de la estructura del programa y escritura del texto del presentador; Realización, planificación de la dotación técnica, desglose de planos para desarrollar el formato en función de cada sección; Equipo técnico (cámara, sonido, iluminación...), que grabará el formato; Promoción, creación de la estrategia comercial del programa para conseguir la máxima difusión; Creatividad, desarrollo de la identidad de marca del programa y de los soportes publicitarios, y Redes, gestión de perfiles sociales para dar visibilidad al producto y ampliar su impacto en la audiencia, así como promover la conversación social.

Conseguir la atención del alumno y mantenerla, empezar con el estudiante en lugar de con la disciplina, situarle en el centro del aprendizaje y ubicar una meta real que les interese, por ejemplo, la adquisición de habilidades profesionales.

En relación con ello, cabe apuntar que la atención estaba asegurada porque la idea de practicar las competencias profesionales sobre este tipo de formato vino, precisamente, por los alumnos. Además, pronto entendieron que no sólo ponían en prácticas las competencias profesionales propias de cada departamento sino también habilidades transversales como el trabajo en equipo, el liderazgo, la imaginación, organización y toma de decisiones y, luego, en el caso concreto de la tercera edición, el desarrollo de competencias digitales relacionadas con el trabajo en remoto por las circunstancias impuestas por la COVID-19.

Sobre las competencias profesionales de cada departamento, podemos apuntar las siguientes:

» Dirección: concebir la idea del proyecto, establecer el tono del programa, el concepto visual y la estructura, comunicar al resto de equipos las metas a alcanzar, aglutinar las acciones de todos para seguir un mismo hilo argumental y velar por el desarrollo del formato. 
» Producción: gestionar los recursos humanos, técnicos y presupuestarios para ejecutar el programa, controlar la logística de la grabación.

" Guion: establecer la escaleta del programa teniendo en cuenta sus secciones, así como la dinámica del concurso; escribir el guion literario, establecer reuniones y ensayos con los presentadores para adaptar timing e interpretación.

» Realización: elaborar la escaleta técnica junto con el director para conseguir trasladar la idea a la imagen, decidir la tipología de planos, disponer la posición y orden de cámaras en consonancia con las personas que intervengan en el programa, determinar qué plano aparece en pantalla para dar coherencia a la emisión, cuidar los contratiempos técnicos.

» Equipo técnico: grabar, sonorizar, iluminar.

» Promoción: idear la estrategia comercial, impulsar el visionado de la audiencia, seleccionar el mix de medios que se utilizará en la campaña.

Creatividad: diseñar la estrategia creativa a partir de las indicaciones comerciales, definir la imagen de marca y su grafismo, así como del resto de soportes utilizados para establecer una identidad única.

» Redes: crear los perfiles sociales en función de su target, gestionar las cuentas, establecer un plan de comunicación y crear contenido para los diferentes canales que propicie engagement con la audiencia.

Buscar compromisos de trabajo dentro y fuera del aula y convencer a los estudiantes de que la acción lleva a un aprendizaje efectivo.

Trabajar por proyectos convierte al docente en un guía de aprendizaje. La relación es mucho más cercana lo que potencia el compromiso. Un proyecto de es- tas características requiere trabajo fuera del aula y, para ello, la motivación es una clave. Los docentes establecen un clima de trabajo agradable y funcional y recuerdan la importancia de experimentar la profesión en el entorno universitario para estar más preparados en el desarrollo laboral real.

Bajo este esquema se diseña el primer Nebrija Talent, que se ajusta en la segunda edición para dar cabida a un mayor número de estudiantes, movidos por el éxito del programa. Además, el segundo show sirve para evaluar el ejercicio anterior, atender posibles carencias y reforzar el resultado final. De hecho, su dimensión fue mayor, ya que trasladó su localización al salón de actos de la Universidad, lo que conllevó la adaptación técnica y artística de un gran espacio, y amplió la participación de los alumnos en el casting de talentos ya que ahora podían presentarse los estudiantes del resto de facultades.

El primer programa se desarrolló con 15 alumnos de tres grados (Periodismo, Comunicación Audiovisual y Publicidad). La segunda edición contó con 25 y se sumaron los Grados en Comunicación Corporativa y Marketing. La tercera, adaptada al formato telepresencial, aglutinó los esfuerzos de 37 alumnos y la participación de 19 concursantes. Se logró la colaboración de 13 grados ya que a los expuestos se sumó Diseño Digital, Artes Escénicas y Diseño de Moda, dentro de los equipos de trabajo, y en cuanto a la candidatura de concursantes, además de los grados mencionados, Derecho, Educación, Arquitectura y Fisioterapia.

\subsubsection{Organización, planificación y desarrollo}

Se siguieron estas fases:

» Selección del director y jefes de departamento. Esta decisión se toma -por parte de los docentes- en función del curso, experiencia y competencias del alumnado, así como la preferencia de los estudiantes sobre su área de trabajo.

» Ideación del programa, creación del concepto visual y elaboración del guion teniendo en cuenta el propósito narrativo. 
» Exposición por parte del director del concepto al resto de departamentos para marcar las directrices a seguir en consonancia con la idea.

» Casting de talentos y elección de finalistas.

» Elaboración de piezas que se insertarán durante la emisión (vídeos de presentación de los concursantes, spots, jurado...).

» Grabación del programa en formato falso directo.

» Edición del concurso, posproducción de imagen y sonido.

Este proyecto se desarrolla en el segundo semestre español. En marzo se atiende la preproducción, el casting y las piezas audiovisuales. En abril, ensayos técnicos y artísticos; en mayo la grabación y en junio la edición.

\subsubsection{Adaptación a la docencia remota}

El 14 de marzo de 2020, el Gobierno español decreta el estado de alarma y se suspende la docencia presencial. La Universidad Nebrija, experimentada en el desarrollo de programas a distancia, logró desde el primer día adaptar las sesiones académicas al formato telepresencial, gracias a la utilización del sistema de videoconferencia BlackBoard Ultra, anclado en el campus virtual, y a partir de un ágil programa de formación.

Los docentes decidieron seguir con las actividades extra-académicas. Realizar el talent show en formato online suponía un reto pero también una motivación extra.

Se estableció una reunión inicial para que los equipos propusieran ideas para adaptar sus funciones a la situación de pandemia, incentivando su creatividad, la resolución de conflictos y situando al alumno en el centro del proyecto.

La primera acción que se adaptó al formato online fue la realización del casting. Si antes se hacía de forma presencial y se grababa por parte del equipo técnico, ahora serían los concursantes quienes se grabarían una actuación de un minuto para redes. Se deter- minó que la votación de finalistas fuera pública y pasaron a la final los 10 candidatos más votados.

La segunda decisión fue emitir el programa en directo, ya que no podíamos grabar físicamente el show. Se emitió en streaming el 8 de mayo de 2020 y simultáneamente a través de BBU y Youtube para poder ampliar el impacto del producto y el seguimiento de la audiencia.

Esta medida provocó otras acciones:

» Reconversión de los departamentos de Realización, ya que la emisión en streaming no permitía un planteamiento multicámara, y del equipo técnico ante la imposibilidad de una grabación real. Los alumnos fueron distribuidos en otras áreas.

» Refuerzo del departamento de Redes ya que no solo debían publicitar el formato, sino que ahora debían motivar el voto del espectador tanto en el casting como en el resultado final ya que, por primera vez, el ganador fue designado por la audiencia. Asimismo, al ser en directo, el departamento -en unión con el equipo de Promoción y Creatividad- debía impulsar el visionado. Se apostó por la actuación de un artista consagrado al programa -Fran Perea- lo que potenció el movimiento fan.

\subsubsection{Herramientas tecnológicas utilizadas}

Cada fase requirió el uso de diferentes herramientas.

Gráfico 1. Herramientas digitales incluidas en el proyecto de innovación docente

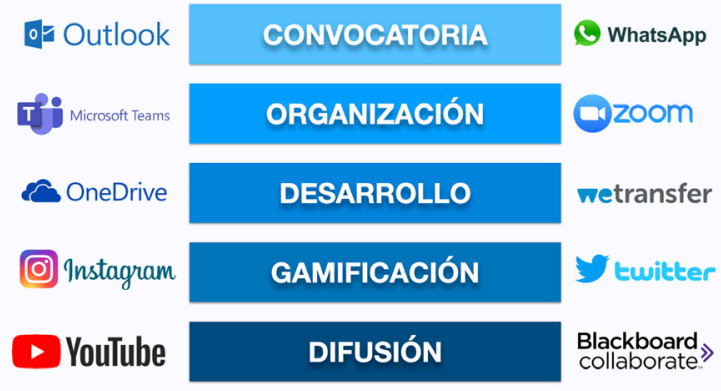

Fuente: Elaboración propia. 
Justificaremos a continuación su uso:

»El email institucional no resultó del todo operativo por lo que se establecieron grupos de whatsapp para una interacción más directa.

» La herramienta Teams está contenida en el paquete Office 365 contratado por la Universidad tanto para alumnos como profesores lo que favorecía su utilización. Además, permite calendarizar reuniones y establecer grupos de trabajo. Tuvimos que añadir la herramienta Zoom para las reuniones con colaboradores externos.

» One Drive y Wetransfer facilitaron, en el primer caso, la puesta en común de documentos de trabajo y la escritura compartida del guion, y en el segundo, el envío de archivos audiovisuales.

» Las redes sociales seleccionadas fueron Instagram y Twitter.

» Como se ha explicado, BBU es la herramienta de videoconferencia anclada en el campus virtual lo que favorece el conocimiento y uso de los alumnos y Youtube nos permitía una difusión mayor.

\subsubsection{Evaluación}

Salvo los cambios en la estructura de trabajo que hemos planteado, el ABP se adaptó muy bien al entorno virtual e, incluso, las herramientas digitales de comunicación favorecieron la interacción.

Evidentemente, los roles profesionales que quedaron más desdibujados fueron los de carácter técnico, apegados a la propia grabación del formato, pero se desarrollaron otras áreas con mayor fuerza en concordancia con lo que ya está pasando en la industria audiovisual en la que agentes como Youtube, Twich o Clubhouse potencian la emisión en directo y sin edición.

El resultado final fue muy satisfactorio y, además de alcanzarse los resultados de aprendizaje, el pro- grama supuso un hito en la comunicación de la Facultad ya que la emisión en streaming y la actuación en redes sociales provocó el conocimiento del formato más allá del propio centro y favoreció que el talent se convirtiera en un escaparate del trabajo de los alumnos. De hecho, fue el primer talent show online de España.

La emisión logró 900 visualizaciones en directo, 3.000 comentarios durante la gala y 75.000 impactos en Instagram y Twitter.

La utilización de herramientas de comunicación digital mejoró la colaboración de alumnos procedentes de diferentes carreras, fortaleciendo la interdisciplinariedad. Este ingrediente es clave ya que en la industria informativa y de entretenimiento se trabaja en equipos multidisciplinares y, así, esta práctica sirve de entrenamiento para futuras situaciones reales.

El proyecto consiguió ser interdisciplinar, también, por la colaboración del resto de laboratorios de la plataforma ya que los alumnos de Nebrija Medialab pertenecen a diferentes titulaciones e, incluso, facultades.

Los estudiantes del área de Actualidad (mayoritariamente del Grado en Periodismo) realizaron la cobertura del talent para la revista [N], los estudiantes de radio (sobre todo del Grado en Audiovisual y de Periodismo) entrevistaron a los finalistas en el programa semanal -ejecutado como podcast durante el confinamiento-, los compañeros de Diseño (principalmente de Diseño Digital, perteneciente al Departamento de Arte) intervinieron en la identidad gráfica y el ámbito de la Publicidad creó cuñas promocionales (a partir del trabajo de alumnos de los grados en Publicidad, Relaciones Públicas, Corporativa, Protocolo, Organización de Eventos y Marketing, éste último del Departamento de Economía).

\subsection{Valoración del alumnado y alcance de los resultados de aprendizaje}

Procedemos al focus group, ya que consideramos que los verdaderos valedores del proyecto son los propios estudiantes. 
Gráfico 2. Resumen de la tercera edición de Nebrija Talent
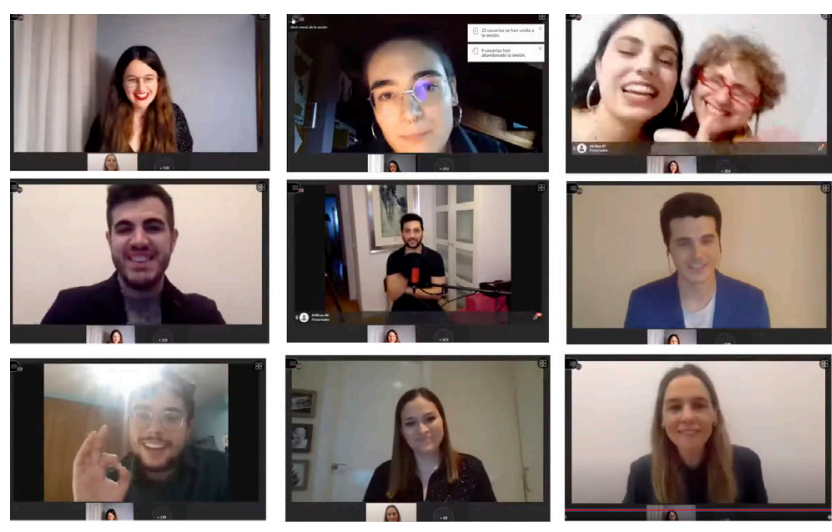

Fuente: Elaboración propia a partir de la emisión del 8 de mayo de 2020 (vía Youtube y BBU)

Los alumnos consideran que esta actividad favorece el desarrollo de sus competencias profesionales. Dentro de las habilidades específicas destacan "todas aquellas referentes a asignaturas como dirección audiovisual, producción, guion, géneros de no ficción, narrativa transmedia y diseño gráfico", indicó el E1. "Luego, aunque el programa no se hiciera de manera convencional, en un plató, y no se pudieran desarrollar los roles técnicos, como hicimos piezas de apoyo para redes sociales, aplicamos conocimientos de cámara, edición, sonido, postproducción... la verdad es que creo que aplicamos todas las competencias", completa.

Además de alumnos de Audiovisual, participaron otros estudiantes. Por ejemplo, la E3, de Periodismo y responsable de la presentación del programa cuenta: "Sentí la experiencia real de conducir un programa en directo, poniendo en práctica competencias de redacción y presentación". "Me sentí igual de nerviosa que si estuviera en un plató real, aunque estaba en el salón de mi casa. Eché de menos al equipo, pero sentía a mis compañeros en cada whatsapp".

Por su parte, la E4, de Artes Escénicas, que actúo como miembro del jurado porque había ganado la edición anterior, expresó: "Me puse del otro lado y me sirvió mucho para reflexionar y saber qué debía mejorar en mis actuaciones y qué perfiles se buscan en televisión”.
En relación con las competencias transversales, todos coincidieron en que había mejorado su capacidad de organización, innovación y creatividad. Destacaron el trabajo conjunto: "A pesar de no ser un proyecto físico, no se sentía que no hubiera equipo" (E1).

Para evaluar la adaptación del proyecto académico a la situación impuesta por la COVID-19, se han considerado los testimonios de aquellos alumnos que han participado en más de una edición.

E7 valora positivamente la adaptación. "Al principio nos sorprendió porque pensábamos que se suspendería, pero luego le dimos la vuelta y pensamos que sería una oportunidad". "Parte del trabajo en redes estaba hecho y fue una clave muy importante desarrollarlas más y dar cabida, por ejemplo, a la fase de casting y a la votación en directo", añade.

E2 manifiesta: "En un contexto en el que era difícil llevar a cabo un talent, buscamos una respuesta entre todos y fue un orgullo". "No eché nada de menos de ediciones pasadas, este año fue aún más cercano y emotivo; notábamos el calor de la gente".

Asimismo, indica que luego fueron viendo cómo "los programas de las cadenas iban haciendo cosas parecidas" y "fue estupendo ver que realmente seguíamos las mismas decisiones que los profesionales".

Destacan, además, como aspectos positivos precisamente la mejora de la comunicación y "el alcance de una audiencia mayor" (E7). "Hicimos récord", recuerda.

$\mathrm{Y}$, de hecho, reconocen que han adquiridos competencias digitales como la utilización de herramientas de teletrabajo pero, también, acciones profesionales como "la búsqueda de información del impacto de la audiencia social en redes" (E2).

También mencionaron que trabajar en grupos numerosos, en los que las acciones estaban divididas, favoreció el intercambio de ideas, ya fuera "a la hora de depurar el guion definitivo" (E5) o "de obtener ideas originales para publicitar el show" (E6).

Los participantes en el focus solo señalaron aspectos negativos en cuanto a la retransmisión. Así, la E4 indicó "errores de sonido entre los participantes 0 cortes derivados de la wifi de cada concursarte". E8 y E9, que solían ejecutar en Medialab roles técnicos como cámara o sonido, comentaron como ya hizo el director del 
programa (E1), que al principio pensaban que sus perfiles quedarían diluidos pero que al grabar piezas con el móvil y editarlas para redes, lograron entrenar sus competencias.

En cuanto a la aplicación de metodologías activas de enseñanza, se muestran positivos y reconocen que fue uno de los motores para matricularse en esta Facultad, donde se sigue el lema "learning by doing".

\section{E7 comenta:}

"Entiendo las metodologías activas como un acercamiento real al mundo laboral. Es la forma más efectiva de asentar los conocimientos y suponen una experiencia de enseñanza en directo, pues aprendemos haciendo. Resulta una oportunidad para trabajar en equipo y acercarme a mis compañeros. Suponen una mejora increíble de destrezas intangibles como la creatividad o el pensamiento crítico, puesto que nos enfrentamos a tomas de decisiones reales".

Por último, queremos destacar lo que supuso la participación en Nebrija Talent III para los alumnos durante el confinamiento:

"Fue un soplo de aire fresco. Todos decíamos que eran las mejores reuniones de la semana. El equipo fue capaz de reinventarse y fue muy emocionante, al ser en streaming, poder compartirlo con la familia. Mis padres lo vieron muy profesional y me sentí orgulloso de que entendieran nuestro trabajo" (E2).

"Una experiencia muy enriquecedora, una práctica muy real. Aprendimos a la vez que nos divertimos y psicológicamente fue muy positivo. No sentía que estaba en un proyecto de clase, parecía más bien que tenía un trabajo y que teníamos que sacar el programa adelante, sacando las castañas del fuego" (E10).
"Fue mi vía de escape durante varias semanas, ya que llegó en el que podría ser mi peor momento durante el confinamiento y eso hizo que tuviese la mente ocupada. Siempre voy a estar agradecida" (E3).

De este modo, y como anuncian autores como Martí (2010) o Johnson, Johnson y Smith (2006), este tipo de proyectos suponen para el claustro una inyección de motivación.

\section{CONCLUSIONES}

1. Nebrija Talent III demostró capacidad de adaptación a las circunstancias provocadas por la pandemia mundial y el confinamiento. Permitió el mantenimiento del calendario de actividades del Laboratorio de Televisión de la plataforma Nebrija Medialab. Así, no se paralizó la formación de los alumnos e, incluso, ampliaron los resultados de aprendizaje previstos con un aumento de la creatividad, el trabajo en equipo, la experimentación con las nuevas tecnologías y el trabajo colaborativo en red.

2. La metodología ABP resultó adecuada y, de hecho, se adapta bien a este tipo de entornos de aprendizaje siempre que se dispongan de las herramientas digitales necesarias. Se certifica Whatsapp como principal instrumento de comunicación y organización de equipos, Youtube como canal de difusión e Instagram como red social predominante para ampliar el impacto del proyecto y la participación del público universitario.

3. El programa favoreció el sentimiento de comunidad universitaria entre estudiantes de distintas facultades. Fomentó la interacción y la colaboración interdisciplinar. Se convirtió en un escaparate de los proyectos de innovación docente de la Facultad ya sea para la comunidad universitaria, para los alumnos interesados en 
estudiar en Nebrija, para los paneles de renovación de las acreditaciones que evidencian claramente el alcance de los resultados de aprendizaje o para la sociedad en general.

4. A nivel de comunicación, mejoró el posicionamiento de la Facultad en RRSS con la interacción de los propios alumnos y se logró visibilizar los proyectos del centro no solo a través de redes sociales propias, sino también de los colaboradores y los propios alumnos que, al sentirse centro y motor del proyecto, favorecieron la difusión del programa.
5. Además de permitirnos seguir ofreciendo una formación experiencial, actualizada, interdisciplinar y adaptada a las necesidades del mercado profesional incluso en el entorno de la $\mathrm{CO}$ VID-19, el proyecto motivó a los alumnos, les ilusionó y endulzó una etapa especialmente dura. Su satisfacción, agradecimiento y sonrisas son el mejor premio de este concurso de talentos. I

\section{REFERENCIAS}

» Bain, Ken (2009). Lo que hacen los mejores profesores. Valencia: PUV.

» Besalú-Casademont, Reinald; Schena, Jucinara; Sánchez-Sánchez, Cristina (2017). Competencias más relevantes en los estudios de Comunicación Audiovisual. Las percepciones de profesionales, docentes y estudiantes. Revista Latina de Comunicación Social, № 72, pp. 1536-1553.

» Canales, Manuel; Peinado, Anselmo (1995): Grupos de discusión. En Delgado, J.M.; Gutiérrez, J. (Eds.). En Métodos y Técnicas cualitativas de investigación en Ciencias Sociales. Madrid: Síntesis.

» Civil i Serra, Marta (2018). Los estudios universitarios en comunicación en España. Análisis de la situación actual para un debate abierto sobre su futuro estratégico. En Postigo, I.; Recoder, M. (coords.). Los y las «tics» en los estudios de comunicación. Málaga: Asociación Española de Universidades con Titulaciones de Información y Comunicación (ATIC), pp. 15-83.

» Fernández-March, Amparo (2006). Metodologías activas para la formación de competencias. Educatio Siglo XXI, 24, pp. 35-56.

» Grijalba, Nicolás; Toledano, Fernando (2014). Nebrija MediaLab: un valor añadido a la docencia y al desarrollo de competencias. Historia y Comunicación Social, 19, pp. 733-745.

» Johnson, David W.; Johnson, Roger. T.; Smith, Karl A. (2006). Active learning: Cooperation in the college classroom (3rd ed). Edina, $\mathrm{MN}$ : Interaction book Company.

» Kapp, Karl M. (2012). The Gamification of Learning and Instruction: Game-Based Methods and Strategies for Training and Education. New York: Pfeiffer.

» Llorens-Largo, Faraón; Villagrá-Arnedo, Carlos; Gallego-Durán, Francisco; Molina-Carmona, Rafael (2021). CoVId-proof: cómo el aprendizaje basado en proyectos ha soportado el confinamiento. Campus Virtuales, 10 (1), 73-88.
» Martí, José A.; et al. (2010). Aprendizaje basado en proyectos. Revista Universidad EAFIT, vol. 46, 158, pp. 11-21.

» Miguel, Begoña (2018). Las competencias digitales en los Grados en Periodismo, Publicidad y Relaciones Públicas y Comunicación Audiovisual en la universidad. El caso de la Comunidad de Madrid. Tesis doctoral, Universidad Complutense de Madrid.

» Perlado, Marta; Saavedra, Marta; Rubio, Juana (2016). Entrenamiento de competencias profesionales en el ámbito de la Comunicación: el caso de Nebrija Medialab. Revista Espéculo, Número 2, pp. 31-45

» Perlado, Marta; Saavedra, Marta (2016). Nebrija Medialab, espacio experiencial de aprendizaje y desarrollo profesional para los estudiantes del área de comunicación. En Membiela, P.; Casado, N.; Cebreiros, M. La docencia universitaria: desafíos y perspectivas. Valencia: Educación Editora, pp. 39-43.

» Rubio, Juana; Perlado, Marta (2015): El fenómeno WhatsApp en el contexto de la comunicación personal: una aproximación a través de los jóvenes universitarios. Revista ICONO14, 13(2), pp. 73-94.

» Saavedra Llamas, Marta; Herrero de la Fuente, Mercedes; Castillo Lozano, Eduardo (2020). La formación en periodismo de datos en España: radiografía de la oferta académica universitaria. Anàlisi: Quaderns de Comunicació i Cultura, 62, 93-109.

» Saavedra Llamas, Marta; Grijalba de la Calle, Nicolás; Pedrero Esteban, Luis Miguel (2018). Hacia una redefinición de las competencias y perfiles profesionales del comunicador audiovisual en el ecosistema digital. Doxa Comunicación, 27, pp. 369-385.

» Sales, Dora; Cuevas-Cerveró, Aurora; Gómez-Hernández, José-Antonio (2020). Perspectives on the information and digital competence of Social Sciences students and faculty before and during lockdown due to Covid-19. El Profesional de la Información, v. 29, n. 4, e290423.

» Sánchez, José (2013). Qué dicen los estudios sobre el Aprendizaje Basado en Proyectos. Actualidad Pedagógica, Número 2013. 